\title{
Prolonged endotracheal intubation: a feasible option for tracheomalacia after retrosternal goitre surgery
}

\author{
Wangang Ren ${ }^{1}$, Xingchen Shang ${ }^{2}$, Honghao Fu ${ }^{1}$, Zhongmin Peng ${ }^{1}$ \\ ${ }^{1}$ Department of Thoracic Surgery, ${ }^{2}$ Department of Breast and Thyroid Surgery, Shandong Provincial Hospital Affiliated to Shandong First Medical \\ University, Jinan, China \\ Contributions: (I) Conception and design: W Ren, Z Peng; (II) Administrative support: Z Peng; (III) Provision of study materials or patients: W \\ Ren, X Shang; (IV) Collection and assembly of data: W Ren, X Shang, H Fu; (V) Data analysis and interpretation: W Ren, X Shang, H Fu; (VI) \\ Manuscript writing: All authors; (VII) Final approval of manuscript: All authors. \\ Correspondence to: Zhongmin Peng. Department of Thoracic Surgery, Shandong Provincial Hospital Affiliated to Shandong First Medical University \\ 9677 Jing 10 Road, Jinan 250000, China. Email: sdslyypzm@163.com.
}

\begin{abstract}
Background: Tracheomalacia may be caused by long-standing compression of retrosternal goitre because of destruction of support of tracheal cartilages. Life-threatening airway collapses may occur after surgical removal of goitre. However, available literature on management methods of tracheomalacia is sparse. Our study highlights prolonged endotracheal intubation as a feasible treatment method.

Methods: This retrospective study analysed 106 thyroidectomies for retrosternal goitre performed between 1994 and 2019. We recorded each patient's clinical profile. Severe tracheomalacia was confirmed through the following: soft and floppy trachea on intra-operative palpation and the collapse of cross-sectional tracheal area measured in computed tomography (CT) images by $>80 \%$. We assessed the extent of airway stenosis of these cases. All severe cases were treated by prolonged endotracheal intubation.

Results: Surgical treatment was successfully performed in all 106 retrosternal goitre patients with no mortality. Seventeen severe tracheomalacia cases were confirmed. The extent of airway stenosis was assessed: the minimal tracheal diameter of compressed trachea was $0.2-0.4$ [mean 0.31, standard deviation (SD) 0.06] $\mathrm{cm}$, and the narrow tracheal length was 4-6.7 (mean 5.1, SD 0.6) $\mathrm{cm}$. These patients underwent endotracheal intubation for $17-47 \mathrm{~h}$ after surgery. All patients were transferred to the general ward after extubation and successfully discharged. There were no cases of tracheal stenosis on follow-up.

Conclusions: Tracheomalacia is a rare but serious complication of retrosternal goitre surgery. Based on our experience, prolonged endotracheal intubation is a feasible treatment for tracheomalacia after retrosternal goitre surgery.
\end{abstract}

Keywords: Prolonged endotracheal intubation; tracheomalacia; retrosternal goitre (RSG)

Submitted Nov 26, 2019. Accepted for publication May 13, 2020.

doi: $10.21037 /$ apm-19-552

View this article at: http://dx.doi.org/10.21037/apm-19-552

\section{Introduction}

Tracheomalacia refers to a condition when cartilaginous rings around the trachea are not strong enough to fully support it. Tracheomalacia is characterised by tracheal collapse in excess of $50 \%$ of the diameter during expiration in spontaneously breathing patients $(1,2)$. It can be either congenital or acquired in normally developed trachea after traumatic insult, external compression, infection, or inflammation (3).

Patients with mild tracheomalacia may be asymptomatic, while others may develop clinical symptoms including cough, stridor, prolonged or recurrent pulmonary infection, difficulty performing exercise, dyspnoea, apnoea, oxygen dependence, and apparent life-threatening events. Expiratory stridor or wheezing is the most common sign on 

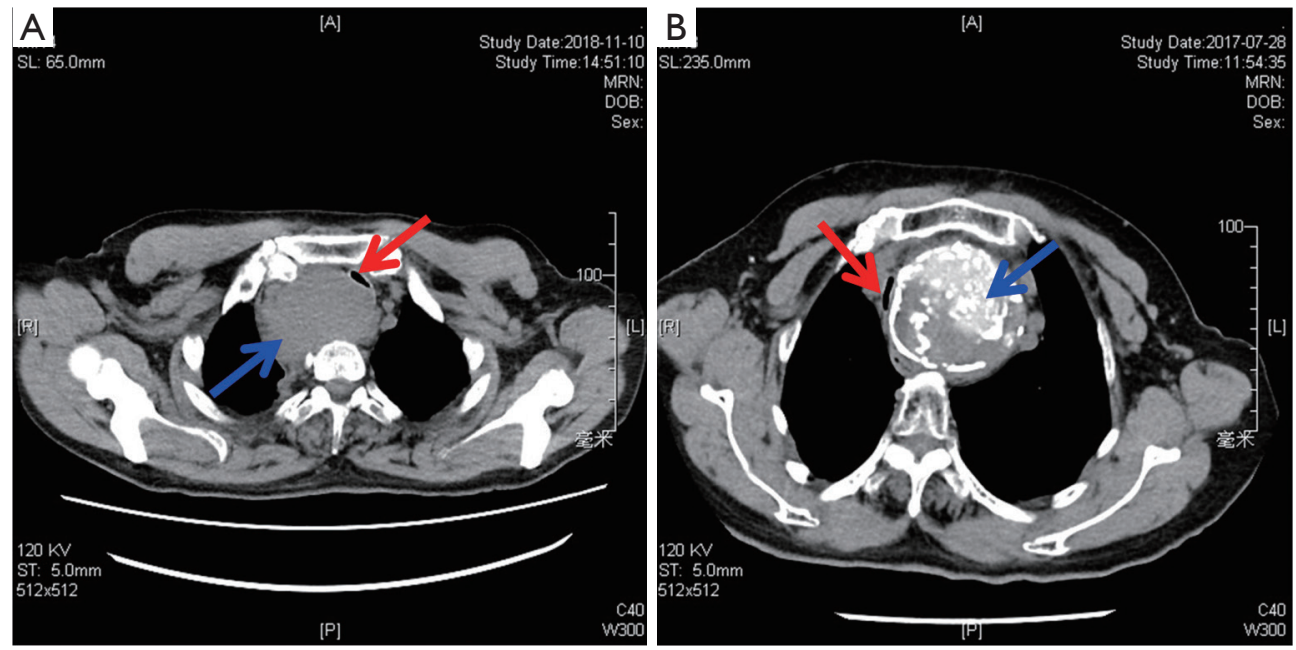

Figure 1 CT images of compressed trachea by retrosternal goitre. (A) and (B) are from different patients. The red arrow points to the compressed and deformed trachea. The blue arrow points to the retrosternal goiter. The goiter in (B) is rich in calcification.

physical examination $(1,4)$.

Tracheomalacia is a serious complication that may occur in patients after retrosternal goitre (RSG) surgery. RSG is clinically and/or radiologically defined as an extension of the thyroid below the sternal notch when the patient is in the supine position (5). The natural history of goitre is one of progressive growth. Long-standing giant goitres may result in weakness of the tracheal wall due to external compression. Certain tracheomalacia cases caused by RSG may cause an emergency with acute airway obstruction in the recovery room or general ward after tracheal extubation $(6,7)$. However, there is a lack of consensus about the optimal treatment of tracheomalacia after RSG surgery. This study examined a single-surgeon series of 17 cases with severe tracheomalacia. These patients were treated with prolonged endotracheal intubation. We describe their clinical features, treatment and outcomes. The purpose of this study was to determine characteristics of these patients; we suggest prolonged endotracheal intubation as a management method of tracheomalacia after RSG surgery.

We present the following article in accordance with the STROBE reporting checklist (available at http://dx.doi. org/10.21037/apm-19-552).

\section{Methods}

We reviewed hospital records of all patients who underwent thyroidectomy for RSG at the Department of Thoracic Surgery, Shandong Provincial Hospital, between May
1994 and May 2019. Most cases were referred form other hospitals. The study was conducted in accordance with the Declaration of Helsinki (as revised in 2013). The study was approved by the Medical Ethics Commission of Shandong Provincial Hospital (No. 2019-214), and all patients signed informed consent.

The state of trachea was assessed by preoperative computed tomography (CT) imaging and intraoperative palpation. Tracheomalacia was determined by intraoperative palpation. Severe tracheomalacia was confirmed through the following: soft and floppy trachea on intraoperative palpation, collapse in the cross-sectional tracheal area measured in CT images $>80 \%$ (Figure 1). For severe tracheomalacia, endotracheal intubation was prolonged in the surgical intensive care unit for about 1-2 days. Ventilator-assisted ventilation was generally not required. Subsequently, the tracheal cannula was extracted and the patient was transferred to the general ward. Based on the data, we recorded each patient's clinical profile, size of goitre, surgical approach, final pathology, and postoperative complications. We also assessed the extent of airway stenosis (minimum tracheal diameter, length of compressed segment) through CT scanning of the neck and thorax. All CT scans were reviewed by an experienced radiologist in thyroid imaging and airway situation.

We recorded the size of RSG by using the cross-section imaging CT system (CSI-CT) classification (8). This classification divides the thyroid extension into three grades according to the position of the inferior goitre border: 
Table 1 Patient characteristics with severe tracheomalacia $(n=17)$

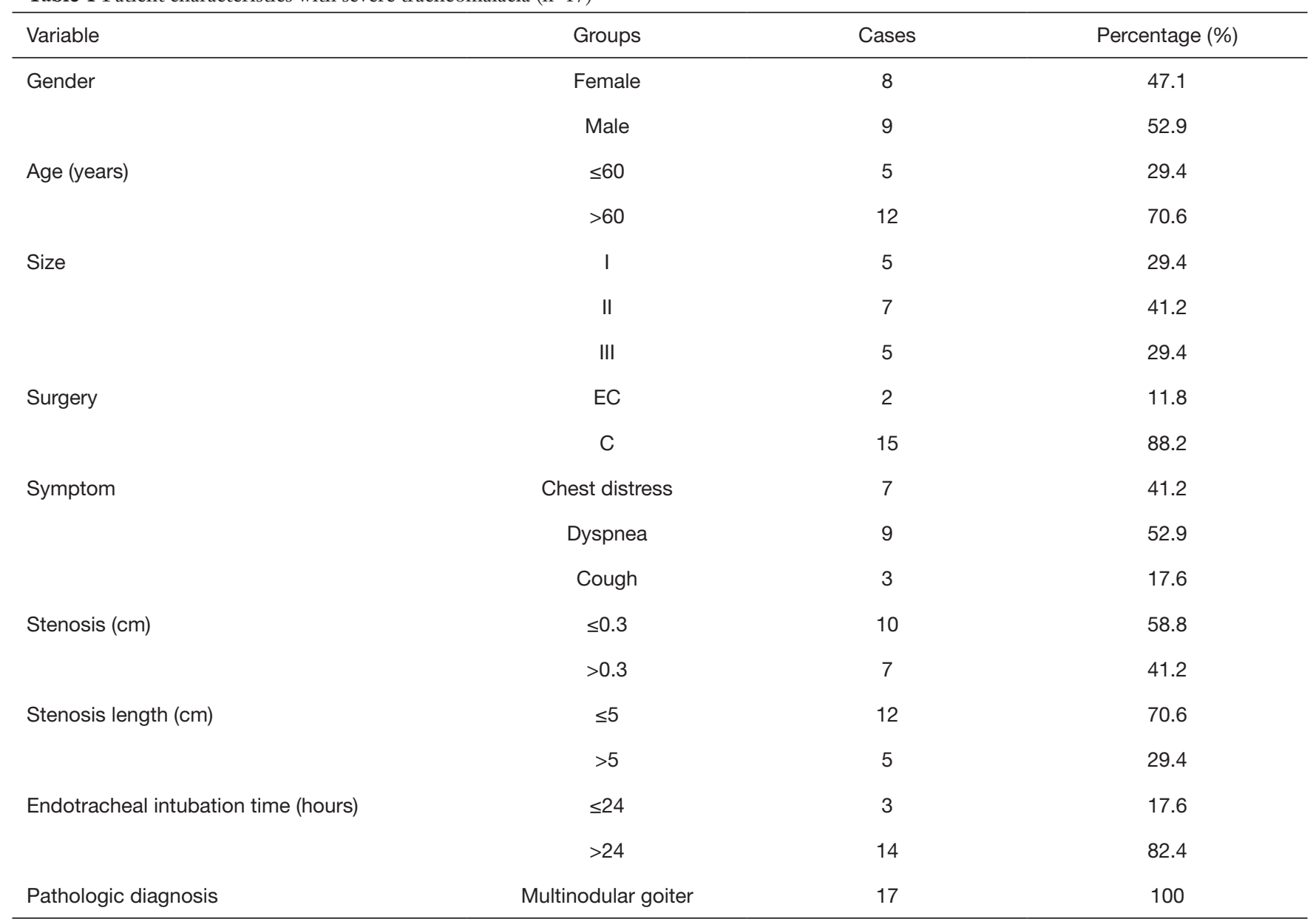

Size (I, II, III) was divided through the cross-section imaging CT system classification. C, cervical approach; EC, ex-cervical approach.

grade I: the inferior limit of the thyroid gland is between the thoracic outlet and the aortic arch; grade II: the inferior goitre border is in between the concavity and convexity of the aortic arch; and grade III: the thyroid extends further down the aortic arch.

Statistical analysis was performed using the SPSS v.16.0 software. Data were analysed as frequency, mean \pm standard deviation (SD).

\section{Results}

Between May 1994 and May 2019, 106 patients underwent thyroidectomy for RSG at the Department of Thoracic Surgery, Shandong Provincial Hospital. Seventeen (16.0\%) patients had severe tracheomalacia.
Clinical features of the 17 patients are presented in Table 1 . The mean age was 64.8 (SD 7.1) years, with a range of $55-78$ years. The female to male ratio was $8: 9$. The most frequent symptom of these patients were dyspnoea, chest distress and cough. Five patients had a grade I RSG goitre, seven patients had grade II, and five patients had grade III, according to the CSI-CT classification. Fifteen patients underwent thyroidectomy through the cervical approach. Partial sternotomy was performed for the other two patients as one had an "iceberg"-shaped goitre and the other patient had a calcified goitre. The minimal tracheal diameter of compressed trachea was $0.2-0.4$ (mean $0.31, \mathrm{SD} 0.06) \mathrm{cm}$, and the narrow tracheal length was 4-6.7 (mean 5.1, SD 0.6) $\mathrm{cm}$. The duration of prolonged endotracheal intubation was $17-47 \mathrm{~h}$. Aetiology of goitre in these 17 patients was 
multinodular goitre. The perioperative death rate was zero. One patient had postoperative pneumonia and recovered after 7 days of anti-inflammatory treatment.

All patients were transferred to the general ward after extraction of the tracheal cannula and successfully discharged. There were no cases of tracheal stenosis during follow-up of 6 months to 3 years.

\section{Discussion}

This study was conducted to determine the feasibility of prolonged endotracheal intubation to manage tracheomalacia after RSG surgery. Despite increasing recognition of tracheomalacia, there remains some uncertainty regarding its identification, causes, and treatment. Most tracheomalacia cases in children are associated with oesophageal atresia or with an anomaly of the aortic arch or the supra-aortic vessels (9). In most cases, if treatment becomes necessary, surgery is preferred to a stent application and aortopexy is the most commonly performed surgical method (10). Acquired or secondary tracheomalacia is caused by the degeneration of normal cartilaginous support from a variety of causes. Longstanding giant goitres can lead to tracheomalacia. Tracheal cartilage may be unable to maintain its patency throughout the breathing cycle after thyroidectomy. During expiration, the intrathoracic pressure rises above the intraluminal pressure of the trachea, and in the absence of cartilaginous support, the trachea collapses $(4,11)$.

The incidence of tracheomalacia has been inconsistent in different reports. Evidence of tracheomalacia secondary to thyroid goitre is largely limited to areas with endemic goitres. In 2008, White et al. performed a systematic literature review regarding surgical treatment of substernal goitres (12). They analysed 12 case series of airway complications after thyroidectomy for substernal goitre. The incidence of tracheomalacia varied, $0 \%$ in seven series, $0.8-2.7 \%$ in four series, $5.7 \%$ in one series and $10.3 \%$ in one series. Agarwal et al. reported a high incidence of tracheomalacia (37.8\%) following thyroidectomy in patients with long-standing large goitres (goitre weight $>400$ g) (6).

There has been no consensus on optimal treatment of tracheomalacia caused by external compression in adults. Treatment is unnecessary in most cases. However, if a patient has evidence of severe tracheomalacia, appropriate treatment of this condition is indicated to prevent catastrophic airway collapse. Treatment options include continuous positive airway pressure, tracheostomy, tracheoplasty, airway stent implantation, tracheal segmentectomy, and so on (13-15). There is no uniform treatment standard, and tracheostomy is the most commonly used treatment nowadays. In a systematic review of available literature, about half of total tracheostomies performed in all series combined were due to tracheomalacia, and about one-third of patients with tracheomalacia had a tracheostomy (12).

According to our experience, even severe tracheomalacia following RSG surgery does not require surgical treatment. Prolonged endotracheal intubation is a feasible option for this condition. There have been a few reports on extending endotracheal intubation. A report by Agarwal et al. cites two cases (6). Chauhan reported two cases, one for $24 \mathrm{~h}$ and another for $48 \mathrm{~h}$ (16). Paul reported a case of endotracheal intubation prolonged for $24 \mathrm{~h}$ before tracheostomy. Fibreoptic bronchoscopic examination revealed that the airway collapsed from two-thirds to onehalf of the anteroposterior diameter after $24 \mathrm{~h}$ of extending endotracheal intubation. The patient's relatives, however, chose tracheostomy over a few more days of intubation (17). In our report, we prolonged endotracheal intubation for 17 $47 \mathrm{~h}$ in 17 patients with severe tracheomalacia. In the severest case, the minimum tracheal diameter was only $0.2 \mathrm{~cm}$ and the length of compressed segment was $5 \mathrm{~cm}$. We did not use continuous positive airway pressure, tracheostomy, or tracheoplasty. All patients had no symptoms of airway stenosis and recovered successfully after extubation. In two cases, we performed tracheal endoscopic examination after extubation and found local softening collapse of the airway wall, which had a limited effect on patency of the airway lumen.

However, our study had several limitations. First, the sample size was small, hence, additional clinical data are needed to verify the duration and safety of prolonged endotracheal intubation. Second, the mechanism of this method is unclear. We suggest the possible mechanism: with support of the tube, the softened trachea activity decreases, and it can harden or stick to surrounding tissues more easily.

We consider prolonged endotracheal intubation to be an effective option for the management of tracheomalacia after RSG surgery. Indwelling endotracheal intubation can prevent life-threatening airway collapse. This method is easy to operate, less invasive and low cost. We suggest that prolonged endotracheal intubation is feasible for 
tracheomalacia caused by other external compression factors.

\section{Conclusions}

Tracheomalacia can be caused by long-standing compression by RSG. On the basis of our experience, prolonged endotracheal intubation is a feasible treatment method for severe tracheomalacia after RSG surgery.

\section{Acknowledgments}

Funding: This work was supported by the National Natural Science Foundation of Shandong province (grant number ZR2019BH072).

\section{Footnote}

Reporting Checklist: The authors have completed the STROBE reporting checklist. Available at http://dx.doi. org/10.21037/apm-19-552

Data Sharing Statement: Available at http://dx.doi. org/10.21037/apm-19-552

Conflicts of Interest: All authors have completed the ICMJE uniform disclosure form (available at http://dx.doi. org/10.21037/apm-19-552). The authors have no conflicts of interest to declare.

Ethical Statement: The authors are accountable for all aspects of the work in ensuring that questions related to the accuracy or integrity of any part of the work are appropriately investigated and resolved. The study was conducted in accordance with the Declaration of Helsinki (as revised in 2013). The study was approved by the Medical Ethics Commission of Shandong Provincial Hospital (No. 2019-214). Written informed consent was obtained from the patient for publication of this study and any accompanying images. A copy of the written consent is available for review by the Editor-in-Chief of this journal. Each patient data is retrieved from hospital medical record system. The patient's personal data have been secured.

Open Access Statement: This is an Open Access article distributed in accordance with the Creative Commons Attribution-NonCommercial-NoDerivs 4.0 International License (CC BY-NC-ND 4.0), which permits the non- commercial replication and distribution of the article with the strict proviso that no changes or edits are made and the original work is properly cited (including links to both the formal publication through the relevant DOI and the license). See: https://creativecommons.org/licenses/by-nc-nd/4.0/.

\section{References}

1. Kugler C, Stanzel F. Tracheomalacia. Thoracic Surgery Clinics 2014;24:51-8.

2. Jokinen K, Palva T, Sutinen S, Nuutinen J. Acquired tracheobronchomalacia. Ann Clin Res 1977;9:52-7.

3. Hysinger EB. Laryngomalacia, Tracheomalacia and Bronchomalacia. Curr Probl Pediatr Adolesc Health Care 2018;48:113-8.

4. Carden KA, Boiselle PM, Waltz DA, et al. Tracheomalacia and tracheobronchomalacia in children and adults: an indepth review. Chest 2005;127:984-1005.

5. Hedayati N, McHenry CR. The clinical presentation and operative management of nodular and diffuse substernal thyroid disease. Am Surg 2002;68:245-51; discussion 251-2.

6. Agarwal A, Agarwal S, Tewari P, et al. Clinicopathological profile, airway management, and outcome in huge multinodular goiters: an institutional experience from an endemic goiter region. World J Surg 2012;36:755-60.

7. Agarwal A, Mishra AK, Gupta SK, et al. High Incidence of Tracheomalacia in Longstanding Goiters: Experience From an Endemic Goiter Region. World J Surg 2007;31:832-7.

8. Mercante G, Gabrielli E, Pedroni C, et al. CT crosssectional imaging classification system for substernal goiter based on risk factors for an extracervical surgical approach. Head Neck 2011;33:792-9.

9. Torre M, Carlucci M, Speggiorin S, et al. Aortopexy for the treatment of tracheomalacia in children: review of the literature. Ital J Pediatr 2012;38:62.

10. Valerie EP, Durrant AC, Forte V, et al. A decade of using intraluminal tracheal/bronchial stents in the management of tracheomalacia and/or bronchomalacia: is it better than aortopexy?. J Pediatr Surg 2005;40:904-7.

11. Kandaswamy C, Balasubramanian V. Review of adult tracheomalacia and its relationship with chronic obstructive pulmonary disease. Curr Opin Pulm Med 2009;15:113-9.

12. White ML, Doherty GM, Gauger PG. Evidence-based surgical management of substernal goiter. World J Surg 2008;32:1285-300.

13. Xu S, Zhu J, Zhao G, et al. Tracheal suspension with 
autogenous rib cartilage in a patient with severe tracheomalacia. J Cardiothorac Surg 2019;14:21.

14. Jin S, Bao W. Huge thyroid adenoma with tracheomalacia. Clin Case Rep 2018;6:1635-6.

15. Choo EM, Seaman JC, Musani AI. Tracheomalacia/ Tracheobronchomalacia and hyperdynamic airway collapse. Immunol Allergy Clin North Am 2013;33:23-34.
16. Chauhan A, Serpell JW. Thyroidectomy is safe and effective for retrosternal goitre. ANZ J Surg 2006;76:238-42.

17. Paul M, Kannaujia A, Chatterjee A, et al.. Serial fiber optic bronchoscopy (FOB) to predict the need of tracheostomy in tracheomalacia after thyroidectomy in long standing goiter. J Clin Anesth 2018;47:9-10.
Cite this article as: Ren W, Shang X, Fu H, Peng Z. Prolonged endotracheal intubation: a feasible option for tracheomalacia after retrosternal goitre surgery. Ann Palliat Med 2020;9(4):1764-1769. doi: 10.21037/apm-19-552 